P47

OUTCOMES OF STATIC VS CONTINUOUS ROLLING COMMUNITY PULMONARY REHABILITATION (PR) PROGRAMMES

doi:10.1136/thx.2010.150961.47

JL Tomkinson, M Rossdale. Bristol Community Health, Bristol, Bristol

Introduction \& objectives Previous research in PR has focussed on duration and location of courses, and models of exercise therapy. In order to meet a commissioning adherence target for 2009-2010, the Bristol community PR team modified stand alone static programmes (SP) (6week) seeing 150 people per year to add in a continuous rolling programme (CRP) (2-4 new patients start every week attending 6 weeks) with the aim of improving adherence to programmes. The objective of this study was to examine if the outcomes of a CRP were as effective as the SP.

Method Retrospective analysis of outcome data for year 2009-2010 for improvement in $6 \mathrm{~min}$ walk test $(\mathrm{m})$, change in Chronic Respiratory Disease Questionnaire (CRDQ), and attendance. Patients were offered a choice of programme at their initial clinic assessment.

Results Results for the two groups are shown in Abstract P47 Table 1. There were no significant differences between groups at baseline. The CRP had 55 (174\%) more patients complete the programme throughout the year. There was no significant difference between groups for improvement in walking distance, attendance or CRDQ-D, CRDQ-F or CRDQ-EF. There was a significant improvement in the CRDQ-M in the CRP group.

Abstract P47 Table 1 Comparison data for static and rolling programmes 2009-2010

\begin{tabular}{lccl}
\hline & $\begin{array}{l}\text { Static } \\
\text { programme } \\
(\mathbf{n}=\mathbf{1 0 0 )}\end{array}$ & $\begin{array}{l}\text { Continuous rolling } \\
\text { programme } \\
(\mathbf{n}=\mathbf{1 7 4 )}\end{array}$ & p-value \\
\hline Change in 6MWT (m) & $119(92.4)$ & $102(87.5)$ & 0.42 \\
Mean (SD) change in CRDQ-D & $0.98(1.07)$ & $0.99(1.12)$ & 0.76 \\
Mean (SD) change in CRDQ-F & $0.93(1.15)$ & $0.90(1.01)$ & 0.70 \\
Mean (SD) change in CRDQ-EF & $0.57(1.07)$ & $0.78(1.16)$ & 0.15 \\
Mean (SD) change in CRDQ-M & $0.72(1.01)$ & $0.97(1.02)$ & $0.05^{*}$ \\
Mean (SD) attendance & $8(4.2)$ & $8.5(4.3)$ & 0.37 \\
Number of patients attending & $72(72)$ & $127(73)$ & \\
8/12 sessions (completers) (\%) & & &
\end{tabular}

6MWT. 6 min walk test; CRDQ: Chronic Respiratory Questionnaire, self-reported, D-Dyspnoea, F-Fatigue, EF-Emotional Function, M-Mastery: Attendance out of 12 possible sessions.

Conclusion This audit shows that the outcomes for a CRP are as good as SP and can be used as an effective method of delivering PR. Both groups require the same amount of staff time; however the CRP saw more patients and had more completers. This may be as cohorts of COPD patients often lose members which can reduce SP's by up to $33 \%$. Within a CRP as patients drop out, new patients can be started keeping the cohort at a set level, thus allowing more patients to be seen. There appears to be a positive effect on CRDQ mastery with patients scoring significantly higher on this in the CRP. This may be an effect of patients who have attended for a while taking on the role as 'experienced buddy' helping the naive patient (Heisler 2009), however further research to investigate this is warranted.

\section{Pulmonary rehabilitation \\ P48 ATTENDANCE RATES AND RESPONSE TO PULMONARY REHABILITATION}

doi:10.1136/thx.2010.150961.48

CM Nolan, KA Ingram, RP Fowler, AL Clark, WD Man. Harefield Pulmonary Rehabilitation Team and Biomedical Research Unit, Royal Brompton and Harefield NHS Foundation Trust, Harefield, Middlesex, UK

Background Although randomised controlled trials and meta-analyses have demonstrated that pulmonary rehabilitation (PR) improves exercise capacity, there is a variability of response and a proportion of PR participants have not shown clincally significant improvements. We hypothesised that attendance record is an important determinant of exercise response to PR

Methods 116 consecutive participants, who attended and completed an outpatient PR programme between September 2009 and June 2010, were included in the study. Subjects attended supervised PR twice per week for 8 weeks, with daily unsupervised home exercise. 107 subjects had pre- and post-PR data. Attendace rate at the supervised sessions was recorded, and good attendance was defined a priori as $75 \%$ or more attendance. Changes in incremental shuttle walk (ISW) pre- and post-PR were calculated for good and poor attendance groups and compared using Mann-Whitney test. Spearman rank correlation and univariate regression was used to assess the relationship between attendance rate and change in ISW following PR.

Results Results were expressed as mean (SD) or median (25th 27th centiles). Patient baseline characteristics were as follows: $55 \mathrm{M}$ 52F, age: 69 (63, 76), FEV1: 1.28 (0.85, 1.89), MRC dyspnoea score; 3 (3, 4), BMI: 27.4 (23.7, 32.1) and ISW: 180 (80, 320). Mean attendance rate was $83(17) \%$. There was no significant difference in age, MRC dyspnoea score and ISW between good and poor attendance groups. Median change in ISW following PR was significantly better in the good attendance group compared with the poor attendance group ( 60 vs $10 \mathrm{~m} ;<0.02$ ). Although Spearman rank correlation was significant (Spearman $r=0.21 ; p=0.03$ ), there was no significant linear relationship $(\mathrm{r} 2=0.01 ; \mathrm{p}=0.35)$ between attendance rate and change in ISW following PR.

Discussion Patients attending more than 75\% of PR sessions improve ISW distance in response to PR more than those attending less than $75 \%$ although the effects of confounding factors cannot be excluded. There is no linear relationship between attendance rate and improvement in ISW. There are likely to be multiple factors determining response to $\mathrm{PR}$.

\section{P49 SEASONAL EFFECT UPON ATTENDANCE AT A PULMONARY REHABILITATION PROGRAMME}

doi:10.1136/thx.2010.150961.49

GE Walker, C Lee, SL Elkin. Chest and Allergy Department, St Mary's Hospital, Imperial College Healthcare NHS Trust, London, UK

Introduction Pulmonary rehabilitation (PR) programmes have been shown to reduce symptoms and improve exercise tolerance in COPD. Improving rates of attendance at PR sessions is vital to ensuring a cost-effective service. The role of seasonality in attendance at PR programmes has not previously been evaluated.

Methods Attendance rates, between 2007 and 2010, at a PR programme in one London borough were reviewed. The number of patients attending assessment and subsequent bi weekly PR sessions 
was examined and demographic information collected. The data were divided by season - winter months incorporating October to March and summer months, April to September. The null hypothesis that there would be no difference between attendance rates in summer months compared to winter months was tested using a Mann-Whitney $U$ test. The attendance data were correlated, using Pearson's product-moment correlation coefficient, with local monthly weather data, obtained from the Met Office, for temperature, hours of sunshine and rainfall over the time period.

Results In total 506 assessment appointments were made to achieve 258 attendances, an attendance rate of $51 \%$. For assessments, there was no difference in attendance between the winter and summer months (50 and 51\% respectively). For the group PR sessions, 2325 appointments were made to achieve 1613 attendances (69\%). The overall attendance rate at group sessions during winter was $64 \%$ compared to $74 \%$ during summer. Non-parametric testing of the data revealed the seasonal difference to be statistically significant $(p<0.05)$. Attendance rates show weak, positive correlation with maximum and minimum temperatures $(r=+0.51,+0.44$ respectively) and sunlight hours $(\mathrm{r}=+0.55)$, and weak negative correlation with amount of rainfall $(\mathrm{r}=-0.33)$.

Conclusion Attendance rates were significantly worse during winter compared to summer. This needs to be taken into account when planning PR services and in local efforts to maximise patient participation. The weak correlation between attendance and specific weather indicators suggests that weather conditions may contribute to this pattern. Confounding patient factors such as illness exacerbation and environmental issues such as transport need to be further evaluated in the context of seasonality to better understand this relationship.

\section{P50 FACILITATION OF CONTINUED EXERCISE VIA PATIENT VOLUNTEERS WITH CHRONIC OBSTRUCTIVE PULMONARY DISEASE (COPD) FOLLOWING A PULMONARY REHABILITATION PROGRAMME: A FEASIBILITY STUDY}

doi:10.1136/thx.2010.150979.1

${ }^{1} \mathrm{C}$ A Langley-Johnson, ${ }^{1} \mathrm{E}$ Jenkin, ${ }^{2} \mathrm{C}$ A E Dyer, ${ }^{3} \mathrm{~K}$ Gruffydd-Jones, ${ }^{4} \mathrm{~N}$ Harris, ${ }^{5} \mathrm{M}$ Reed, ${ }^{5} \mathrm{G}$ Taylor. ${ }^{7}$ Wiltshire PCT, Chippenham, UK; ${ }^{2}$ Royal United Hospital, Bath, UK; ${ }^{3}$ Box Surgery, Chippenham, UK; ${ }^{4}$ Bath Institute of Medical Engineering, Bath, UK; ${ }^{5} B A T H$ University, Bath, UK

Introduction After pulmonary rehabilitation activity levels gradually decline, on average, back to baseline over 12-18 months. The NHS has highlighted the value of peer support for patients with chronic disease, but this has not been fully evaluated in patients with COPD. Therefore we designed an observational study with the aim of exploring and assessing an exercise maintenance programme with peer volunteer support over a 6-month period, in order to inform a future large-scale study.

Methods Patients were recruited from a 7-week outpatient community pulmonary rehabilitation programme. Six peer volunteers with COPD were trained and allocated up to six patients each. A fortnightly exercise maintenance programme was set up in one locality led by a physiotherapist \& assistant. This was supported by a programme of home-based exercise, goal setting and feedback on activity levels. Peer volunteers met with their groups each month to encourage individual activity. The primary outcomes were participation levels and attendance rates; secondary outcomes were activity levels and health status. Qualitative interviews demonstrated the individual variance in exercise habits and motivations see Abstract P50 Table 1.
Abstract P50 Table 1

\begin{tabular}{ll}
\hline Facilitators & Constraints \\
\hline Group providing social destinationSupportive & Group social activity displacing exercise \\
environment & activity \\
Group creating a shared sense of belonging & Personal outlook and life events inhibiting \\
and acceptance enhanced by common goals, & motivation and ability \\
same condition and shared characteristics & to exercise \\
Mentors adoption of counsellor/support role & Exercise programme: Lack of intensity \\
Staff generating trust through caring attitude & Self-directed, no external motivation \\
and expertise & Limited exercise equipment \\
Pre-conditioned to exercisePersonally & Fortnightly sessions \\
motivated & Group vs individual \\
Fostered through attendance at PACE & Sacrificing own needs for group cohesion \\
rehabilitation programme &
\end{tabular}

Results There were 91 potential recruits who completed rehabilitation (13 didn't meet criteria, six became unwell). 37 refused to participate, citing reasons such as poor health, no difficulty in exercising or problems travelling to the centre. Of the 35 that agreed to participate, the attendance rate at exercise was $70 \%$, and 30 completed the 6-month programme. In this group Shuttle Walking Test distances were maintained at 6 months (mean $313.2 \mathrm{~m}$ postrehabilitation compared with $317.8 \mathrm{~m}$ at 6 months, $\mathrm{p}=\mathrm{NS}$ ), and Chronic Respiratory Disease Questionnaire scores were also maintained. Qualitative analysis supported the concept of peer volunteers, while many individuals wanted flexibility and access to a range of activity programmes.

Conclusions This study suggests that maintenance programmes need to be individually tailored rather than prescriptive, in order to maximise uptake and meet the challenges posed by exacerbations. The role of peer volunteers in promoting activity is supported by the qualitative analysis, while exercise classes may help those who wish to attend.

\section{P51 GROUP-BASED PULMONARY REHABILITATION DELIVERED} TO THE HOME VIA THE INTERNET-A FEASIBILITY STUDY

doi:10.1136/thx.2010.150979.2

${ }^{1} \mathrm{D}$ J Godden, ${ }^{2} \mathrm{~J}$ Colligan, ${ }^{3} \mathrm{~A}$ Aitken, ${ }^{3} \mathrm{~A}$ Taylor. ${ }^{1}$ Centre for Rural Health, University of Aberdeen, Inverness, Scotland; ${ }^{2}$ Raigmore Hospital, Inverness, Highland, UK; ${ }^{3}$ Distance Lab, Forres, Scotland

Background Many patients cannot attend group-based pulmonary rehabilitation programmes due to remoteness from the clinic or transport problems. We describe a feasibility study of a novel method of delivering a programme via internet-based videoconferencing.

Methods We re-boxed a mini PC in a simple case with a single large on/off button, which was connected to each patient's home television and wired to the Internet, together with a web cam, speakerphone, and, where appropriate, a wireless pulse oximeter The physiotherapist delivered the programme from a central location equipped with a desktop PC connected to the internet; web cam; speakerphone and 40-in. video screen. When patients turned on their mini PC and TV and switched to the video channel, they automatically joined the class. All patients and the physiotherapist were visible and audible on all TV screens, to create a group spirit. A supporting screen was added to the physiotherapist's set-up to display patient names, exercise duration $(30-60 \mathrm{~s})$, pulse oximeter readings, and an exercise timer. A desktop video conferencing service was employed. A standard pulmonary rehabilitation exercise programme was delivered 